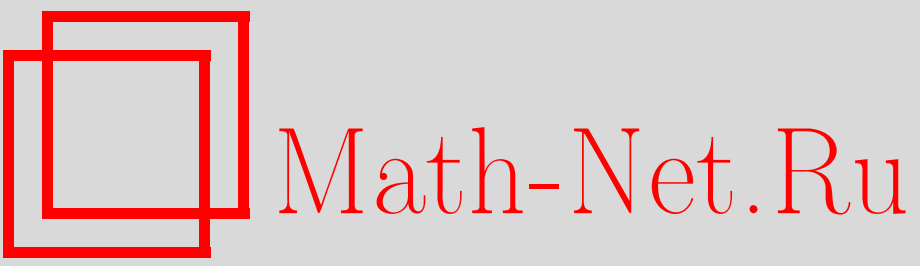

В. В. Белокуров, Ю. П. Соловьев, Е. Т. Шавгулидзе, Теория возмущений со сходящимися рядами для функциональных интегралов по фейнмановской мере, УМH, 1997, том 52, выпуск 2, 155-156

DOI: https://doi.org/10.4213/rm828

Использование Общероссийского математического портала Math-Net.Ru подразумевает, что вы прочитали и согласны с пользовательским соглашением

http: //www.mathnet.ru/rus/agreement

Параметры загрузки:

IP: 44.207 .124 .84

26 апреля 2023 г., 14:11:24 


\title{
ТЕОРИЯ ВОЗМУЩЕНИЙ СО СХОДЯЩИМИСЯ РЯДАМИ ДЛЯ ФУНКЦИОНАЛЬНЫХ ИНТЕГРАЛОВ ПО ФЕЙНМАНОВСКОЙ МЕРЕ
}

\author{
В. В. БЕЛОКУРОв, Ю. П. СоловьЕВ, Е. Т. ШАВГУЛИДЗЕ
}

Заметка посвящена распространению метода вычисления функционалшных интегралов с помощью аппроксимации их сходящимися рядами [1]-[3] на функциональные интегралы по фейнмановским мерам вида

$$
I(g)=\int_{H} F(x) e^{-\beta g P(x)} \Phi_{\alpha, A}(d x) .
$$

Мы будем иметь дело с обобщенными фейнмановскими мерами $\Phi_{\alpha, A}(d x)$ на сепарабельном комплексном гильбертовом пространстве $H$, преобразование Фурье которых задается формулой

$$
\int_{H} e^{i(x, y)} \Phi_{\alpha, A}(d x)=e^{-\frac{\alpha}{2}\left(A^{-1} y, y\right)}, \quad x, y \in H,
$$

где $A: H \rightarrow H$ - самосопряженный положительно определенньй ядерньй оператор и $\alpha \neq 0$ - комплексньй параметр такой, что $\operatorname{Re} \alpha \geqslant 0$. (Определение интеграла по обобщенным мерам см. в [4].) В выражении (1) через $P(x)$ обозначен непрерьвньй однородный полином на $H$ степени $2 m, F(x)$ - непрерывньй функционал, удовлетворяющий условию

$$
|F(x)| \leqslant C \exp \left(\delta\|x\|^{2}\right), \quad 0<\delta<\frac{1}{2} \lambda_{1}^{-1},
$$

где $\lambda_{1}$ - наибольшее собственное значение оператора $A$. Относительно параметров $\beta$ и $g$ полагаем $\beta \in \mathbb{C}, \operatorname{Re} \beta \geqslant 0 ; g \in \mathbb{R}, g \geqslant 0$.

ТЕоремА. Пусть для функционального интеграла (1) по обобщенной фейнмановской мере выполняются сформулированные выше условия. Тогда для любого $\varepsilon, 0<\varepsilon<1$, найдется такое число $R_{0}(\sim \ln (1 / \varepsilon))$, что при $R>R_{0}$ интеграл (1) может быть представлен в виде

$$
I(g)=\sum_{k=0}^{\infty} a_{k}(R) g^{k / m}+\omega(g, R)
$$

əде $|\omega(g, R)|<\varepsilon u$

$$
a_{k}(R)=\frac{(-1)^{k}}{2 \pi(2 k) !} \int_{-R}^{+R}\left(\int_{-\infty}^{+\infty} \exp \left\{-i \rho r-\beta r^{2 m}\right\} d r\right) \rho^{2 k} d \rho \int_{H} F(x) P(x)^{k / m} \Phi_{\alpha, A}(d x) .
$$

При любих положительных $g$ ряд $\sum_{k=0}^{\infty} a_{k}(R) g^{k / m}$ сходится.

СХемА ДОКАЗАТЕЛЬСТВА. Из работы [4] следует, что $I(g)=\lim _{n \rightarrow \infty} I_{n}(g)$, где

$$
I_{n}(g)=Z_{n}^{-1} \int_{H_{n}} F(x) e^{-\frac{\alpha}{2}\left(A^{-1} x, x\right)-\beta g P(x)} d x, \quad Z_{n}=\int_{H_{n}} e^{-\frac{\alpha}{2}\left(A^{-1} x, x\right)} d x,
$$

и $H_{n}$ - подпространство в $H$, натянутое на первые $n$ собственных векторов оператора $A$.

Обозначим через $\widetilde{\varphi}(\rho)$ образ Фурье функции $e^{-r^{2 m}}$ :

$$
\widetilde{\varphi}(\rho)=\frac{1}{2 \pi} \int_{-\infty}^{+\infty} e^{-i \rho r} e^{-\beta r^{2 m}} d r
$$

Работа выполнена при поддержке Российского фонда фундаментальных исследований. 
Тогда

$$
e^{-\beta g P(x)}=\int_{-\infty}^{+\infty} \exp \left\{i g^{1 /(2 m)} \rho Q(x)\right\} \widetilde{\varphi}(\rho) d \rho,
$$

где $Q(x)=P^{1 /(2 m)}(x)-$ однородная функция первого порядка.

Следовательно, интеграл $I_{n}(g)$ имеет вид

$$
I_{n}(g)=Z_{n}^{-1}(\alpha) \int_{H_{n}} F(x) e^{-\frac{\alpha}{2}\left(A^{-1} x, x\right)}\left(\int_{-\infty}^{+\infty} \exp \left\{i g^{1 /(2 m)} \rho Q(x)\right\} \widetilde{\varphi}(\rho) d \rho\right) d x .
$$

Заметим теперь, что выполняется следующее неравенство

$$
\left|e^{-\frac{\alpha}{2}\left(A^{-1} x, x\right)} \exp \left\{i g^{1 /(2 m)} \rho Q(x)\right\} \widetilde{\varphi}(\rho)\right| \leqslant C e^{-\operatorname{Re} \alpha \frac{1}{2}\left(A^{-1} x, x\right)} \exp \left\{-|\rho|^{1+\frac{1}{2 m}}\right\} \widetilde{\varphi}(\rho) \mid .
$$

В резулттате получим

$$
I_{n}(g)=\int_{-\infty}^{+\infty} \tilde{\varphi}(\rho)\left(Z_{n}^{-1}(\alpha) \int_{H_{n}} F(x) e^{-\frac{\alpha}{2}\left(A^{-1} x, x\right)} \exp \left\{i g^{\frac{1}{2 m}} \rho Q(x)\right\} d x\right) d \rho .
$$

С помощью замен переменных интегрирования в интеграле $I_{n}(g)$ функциональньй интеграл $I(g)$ приводится к интегралу по гауссовской мере, для которого утверждение теоремы доказано в работе [2].

\section{СПИСОК ЛИТЕРАТУРЫ}

[1] Белокуров В.В., Соловьев Ю. П., Шавгулидзе Е. Т. // ТМФ. 1996. Т. 109. №1. С. 51-59. [2] Белокуров В. В., Соловьев Ю. П., Шавгулидзе Е. Т. // ТМФ. 1996. T. 109. № 1. C. 60-69. [3] Belokurov V. V., Solovyov Yu. P., Shavgulidze E. T. // Mod. Phys. Lett. A. 1995. V. 10. № 39. Р. 3033-3041. [4] Смолянов О. Г., Шавгулидзе Е. Т. Континуальные интегралы. М.: Изд-во МГУ, 1990.

Московский государственный

Принято редколлегией университет им. М. В. Ломоносова 07.06.1996

E-mail: belokur@rector.msu.su; solo@difgeo.math.msu.su; shav@nw.math.msu.su 